Granular Matter manuscript No.

(will be inserted by the editor)

\author{
A. Karrech - D. Duhamel - G. Bonnet • \\ F. Chevoir - J.-N. Roux - J. Canou • \\ J.-C. Dupla •
}

\title{
A discrete element study of settlement in vibrated granular layers: role of contact loss and acceleration
}

Received: October 25, 2018

\begin{abstract}
This paper deals with the vibration of granular materials due to cyclic external excitation. It highlights the effect of the acceleration on the settlement speed and proves the existence of a relationship between settlement and loss of contacts in partially confined granular materials under vibration. The numerical simulations are carried out using the Molecular Dynamics method, where the discrete elements consist of polygonal grains. The data analyses are conducted based on multivariate autoregressive models to describe the settlement and permanent contacts number with respect to the number of loading cycles.
\end{abstract}

Keywords Granular Materials · Vibration · Contacts $\cdot$ Settlement

\section{Introduction}

Ballast materials of railway platforms exhibit complex behavior under repeated loading. With the increase of vehicle speeds as well as comfort and safety requirements, understanding the dynamics of these materials is becoming a crucial issue. Unlike highly agitated granular materials which can

Address:

Université Paris-Est, Institut Navier, LAMI, Ecole des Ponts, 6 et 8 av Blaise Pascal, Cité Descartes, Champs Sur Marne, 77455 Marne la Vallée Cedex2, France.

Prof D. Duhamel (Corresponding Author):

Tel.: +33164153728 Fax: +33 164153741 E-mail: duhamel@lami.enpc.fr

Prof G. Bonnet:

Université Paris-Est, Laboratoire de Modélisation et Simulation Multi-Echelle, 5 bd Descartes, Champs Sur Marne, 77454 Marne la Vallée Cedex2, France. 
be described with the kinetic theory, ballasted layers are generally subjected to dense flow where the trajectories of grains are correlated and the collisions cannot be considered as randomly distributed in terms of positions and velocities. Therefore, such a statistical approach may be inappropriate to predict the response of railway platforms.

In this research work, the Molecular Dynamics method is used to simulate granular samples made of polygonal grains under vibration. Since its introduction by Cundall and Strack [9], this discrete elements method has proved its viability in describing several mechanisms such as granular materials transport [20], mixing [7], segregation [6], compaction [23] etc. Coupled with the experimental approaches, the MD is now recognized as a fundamental tool to investigate the behavior of dense granular materials. Recently, $\mathrm{Lu}$ and McDowell [19] developed an approach based on the MD to investigate the permanent displacements in granular beds, made of irregular shaped grains, under a single loading cycle. The complex geometry was produced using spherical grains assemblies. Although efficient in terms of grain shapes, this technique neglects the inertial effects of the overlapping regions. The discrete element method was also adopted by Lobo-Guerrero and Vallejo [18] on railway platforms to investigate the effect of grain degradation on granular bed response under cyclic loading. The model took into account the rupture of grains using a criterion based on the loading modes and force intensities. Similar approaches such as the molecular dynamics method was used to simulate the vibration of confined granular material made of polygonal components [2; 25]. In this paper, the target is to relate the permanent settlement in partially confined samples to the loss of contacts using statistical analysis. In the second section, a brief description of the simulation method is suggested. The settlement mechanism which represents the residual displacement under sleeper is described through field cases simulation. In the third section, a causality analysis based on multivariate autoregressive models describing the settlement and contact loss is conducted.

\section{Simulation method}

The sample is composed of polygonal grains which are described with a set of ordered vertices $\left(s_{\alpha, i}\right)_{i \in[1,6 \mid]}$. The positions of the vertices can be evaluated using the distances $r_{1}$ and $r_{2}$ as well as the orientation $\theta$, which are shown in figure 1-(a). These parameters follow uniform bounded distributions: $r_{1} \sim$ $U_{\left[r_{\min }, r_{\max }\right]}, r_{2} \sim U_{\left[0.25 r_{1}, 0.75 r_{1}\right]}$, and $\theta \sim U_{[0 ; 2 \pi]}$.

The interaction between grains takes place when an overlap is detected (Figure 1-b). The dichotomy method is used to calculate the shortest distance between the vertices. This leads to the edges which are candidates for interaction. Among this list of edges, the contact segment which relates the couple of intersection points is obtained. It defines the tangential component, 
$\boldsymbol{t}$, of the contact frame and its perpendicular represents the normal component, $\boldsymbol{n}$. The reference point of the frame, $o$, is defined by the middle of the above mentioned intersection points. Once the contact frame, the overlap, and the velocities are known, the contact forces acting on a grain $\alpha$ can be written as follows:

$$
\begin{aligned}
& F_{n}=F_{n}^{e}-\gamma_{n} v_{n} \\
& F_{t}= \begin{cases}F_{t}^{e}-\gamma_{t} v_{t} & \text { if }\left\|F_{t}\right\| \leq \mu F_{n} \\
\operatorname{sign}\left(F_{t}^{e}\right) \mu F_{n}-\gamma_{t} v_{t} & \text { if }\left\|F_{t}\right\|>\mu F_{n}\end{cases}
\end{aligned}
$$

The interaction forces acting on the particles contain elastic terms; by assuming uniform pressure and shear stresses along the contact surface, it can be shown that these elastic forces are linear: $F_{n}^{e}=k_{n} u_{x}$ and $F_{t}^{e}=k_{t} u_{y}$, where $k_{n}=\frac{E}{c_{n}\left(1-\nu^{2}\right)}$ and $k_{t}=\frac{E}{c_{t}\left(1-\nu^{2}\right)}$. $E$ and $\nu$ are the materials properties (Young modulus and Poisson's ratio) whereas the constants $c_{n}$ and $c_{t}$ can be obtained experimentally using a compression test. The interactions also enclose viscous terms denoted by the phenomenological constants $\gamma_{n, t}$ which can be rewritten as dissipation fraction with respect to the critical damping: $\gamma_{n, t}=\alpha_{n, t} \sqrt{m k_{n, t}}$. Finally, the Coulomb friction is included through the threshold $F_{t} \leq \mu F_{n}$, where $\mu$ is the friction coefficient. At the same time the moments are calculated using the contact frame and the contact forces. The discrete element simulations are carried out with the following parameters: density of grains, $\rho_{p}=2710 \mathrm{~kg} / \mathrm{m}^{3}$, Young modulus, $E=46.9 \mathrm{GPa}$, Poisson's ratio, $\nu=0.25$, friction coefficient, $\mu=0.8$, viscous coefficients, $\alpha_{n}=0.8$ and $\alpha_{t}=0.1$, and grain dimensions $r_{\min }=r_{\max }=5 \mathrm{~mm}$ (unless differently specified). It is worthwhile noticing that the interaction with the wall is described the same way as the interactions between grains.

Using the contact forces between two grains $\alpha$ and $\beta$ at the reference point $o$, it is possible to calculate the moments around the centroid $c_{\alpha}$ using the identity $\boldsymbol{M}_{\boldsymbol{c}_{\boldsymbol{\alpha}}}=\boldsymbol{M}_{\boldsymbol{o}}+\boldsymbol{F}_{\boldsymbol{c}} \times \boldsymbol{o} \boldsymbol{c}_{\boldsymbol{\alpha}}$, where $\boldsymbol{F}_{\boldsymbol{c}}$ is the contact force applied on the grain $\alpha$ as expressed beforehand in equation (1). The procedure is used for each component of the sample. Once the moments and interactions are known, the equations of motion can be integrated with respect to time using a finite difference scheme $[9 ; 15 ; 16]$, according to the Molecular Dynamics method.

\subsection{Sample preparation, loading, and boundary conditions}

At the beginning of the process, the grains are subjected to the gravity field until reaching the full equilibrium. The displacements and rotations are calculated using the predictor-corrector algorithm [1], where the contact forces, the moments and the body forces are taken into consideration. Once the equilibrium is reached, the sample - of width $R=75 \mathrm{~mm}$, height $H=150$ $\mathrm{mm}$ and number of grains 175 - is subjected to a sinusoïdal load of the form 
$F=F_{0}+\Delta F \sin \omega t$, where $\omega$ is the circular frequency, $F_{0}$ is the initial force which is taken equal to $0.5 k N$, and $\Delta F$ is the force amplitude. The frequency and force amplitude vary in such a way that the sleeper covers a wide range of accelerations around $\mathrm{g}$ (gravity). The excitation is applied on the inner half $(0 \leq r \leq 0.5 R)$ and upper end $\left(y_{t=0}=H\right)$ of the sample through a rigid sleeper, unless differently specified. The sides $r=R$ and $y=0$ represent the wall (container), they react to the grains actions as described beforehand. However, at $r=0$, a symmetry condition is simulated by omitting the friction effect (Figure 2-(a)). Henceforth, this loading case will be termed as partially confined configuration. The fully confined configuration corresponds to the case where the excitation is applied on the whole upper end of the sample.

Under repeated loading granular materials undergo large displacements towards free regions. It is interesting to notice that observations of displacement fields and strains showed that granular materials deform because of rearrangements of the packing, rather than contact elasticity [24; 8]. Unlike fully confined samples, where the settlement is mainly due to grain rearrangement and overwork, the mobility of grains in partially confined granular materials is predominant. An experimental work performed by the authors [13; 14] on irregular ballasted samples showed that the acceleration of the sleeper plays a key role on the mobility of grains. In both confinement cases, it has been shown that the settlement increases with the acceleration. In addition, in case of partially confined samples, a sharp increase in terms of settlement speed has been noticed when the acceleration exceeds the gravity. At high level of agitation, the loss of contacts is frequent especially at the critical plane relating the edge of the sleeper to the wall as can be seen in figure (2-b).

\subsection{Settlement in terms of acceleration and loss of contacts}

The simulations conducted herein are performed using polygonal grains in order to produce a shape which is similar to the micro-ballast used in the above mentioned study [13; 14]. The obtained numerical results consist of axial displacement of the sleeper with respect to time. The applied forces are varied from 0.5 to $3 \mathrm{kN}$ and the frequency is varied from 20 to $40 \mathrm{~Hz}$, in such a way that the sleeper covers a wide range of accelerations around the gravity. The grains radii are distributed uniformly from $r_{\min }=5 \mathrm{~mm}$ to $r_{\max }=10 \mathrm{~mm}$. In accordance with the experimental procedure, the settlement under sleeper, $h_{\max }$ is described with a logarithmic law of the form: $h_{\max }(N)=A+B \ln (N)$, with respect to the number of cycles $N$. This logarithmic law seems to be valid for different granular materials under cyclic loading $[3 ; 21 ; 22 ; 26]$ The parameters $A$ and $B$ depend on different physical factors such as the degree of confinement, the applied force, and the frequency. These factors are independent and they can affect individually 
the settlement speed. The experimental result revealed that the acceleration which depends simultaneously on the applied force and frequency is the best explicative quantity in terms of correlation. Moreover, it has been shown that the degree of confinement results in different behaviors in terms of settlement versus acceleration. The simulations conducted herein produce most of the experimental features. It can be seen that the partially confined configurations exhibit a critical transition in terms of settlement speed at around $1.4 \mathrm{~g}$, as can be seen in figure (3). This transition has been observed experimentally [13], it is probably due to the loss of contacts which can not be detected easily with the considered experimental setup. Numerical simulation can provide some more information regarding the history of the granular material texture.

Figure (4) shows the variation of the sleeper displacement as well as the number of contacts with respect to the number of loading cycles, at different frequencies. In these particular cases, the number of cycles is limited to around 25, for clarity, and the applied force amplitude is equivalent to $1.5 \mathrm{kN}$. It can be noticed that the settlement is much higher when the acceleration is beyond the gravity level $(f=40 \mathrm{~Hz})$. On the other hand, it can be seen that the number of contacts follows the exciting force, in terms of oscillations. This means that while cyclically loaded, the granular material exhibits local alternating opening and closure of contacts independently of the acceleration level. However, it can be seen that there is a difference of about $20 \%$ in terms of permanent loss of contacts when the sleeper undergoes high acceleration.

Under cyclic loading of partially confined sample, the grains flow towards less loaded regions as can be seen in figure (2-b). More interestingly, it can be seen in figure (5-a) that during the settlement process, contact openings occur at a specific region between the front of the sleeper and the wall frontier. In the same region there is a loss of density as can be seen in figure (5-b).

This critical and localized behavior can be observed during the settlement process independently of the number of cycles. In the following section, it will be shown that there is a causality relationship between the loss of contacts and the settlement speed.

\section{Causality relationship between contacts loss and settlement speed}

In order to find out a logical relationship between the responses of granular materials under vibration, statistical analysis are necessary since the solution is not analytically determinist. In this section, the objective is to investigate the causality between the settlement velocity and the loss of contacts. Therefore, we adopt Granger's approach [12] which was introduced in econometric in order to forecast possible relationship between discrete time

series. However, this approach can be applied for physical systems providing 
accessible responses with respect to time [5]. The model questions whether the prediction of a given variable is improved by taking into account its own history and the history of another variable. The original model concerns only stationary time signals (time series where the first and second moments are independent of time) or linear (time series with a stationary rate of change). More recently, it has been shown [10] that it is possible to conduct causality testing on non stationary time series if (i) they can be approximated by tendency functions and (ii) the estimation errors are stationary.

\subsection{Causality measurement}

The physical quantities of interest are the settlement under sleeper, $\mathrm{h}(\mathrm{t})$, and the number of permanent contacts, $z(t)$. In order to illustrate the causality analysis, a granular sample consisting of polygonal grains of diorite with an average size of $5 \mathrm{~mm}$ is considered. The applied force in this particular case is of frequency $60 \mathrm{~Hz}$ and amplitude $1 k N$. The time series are extracted from the numerical results by averaging over the loading cycles as follows: $z(n)=<z(t)>=\frac{1}{T} \int_{(n-1) T}^{n T} z(t) d t$ and $h(n)=<h(t)>$, where $\mathrm{T}$ is the loading period (Figure 6). The dynamic relationship between the above mentioned variables are then described with a multivariate autoregressive model of the order $l$ as follows:

$$
\begin{aligned}
& h(i)=h^{r}(i)+\sum_{k=1}^{l} a_{k} h(i-k)+\sum_{k=1}^{l} b_{k} z(i-k)+\epsilon_{h}(i) \\
& z(i)=z^{r}(i)+\sum_{k=1}^{l} c_{k} h(i-k)+\sum_{k=1}^{l} d_{k} z(i-k)+\epsilon_{z}(i)
\end{aligned}
$$

where $h^{r}(i)=\alpha+\beta \operatorname{Ln}(i)$ and $z^{r}(i)=\chi+\gamma i$ are the tendency functions with respect to the number of cycles, and $\epsilon$ are the error terms, which are assumed to be independent.

In accordance with the Granger's method, a comparaison in terms of accuracy is conducted between the above description (2) and the following model:

$$
\begin{aligned}
& h(i)=\hat{h}^{r}(i)+\sum_{k=1}^{l} \hat{a}_{k} h(i-k)+e_{h}(i) \\
& z(i)=\hat{z}^{r}(i)+\sum_{k=1}^{l} \hat{d}_{k} z(i-k)+e_{z}(i)
\end{aligned}
$$

where $\hat{h}^{r}(i)=\hat{\alpha}+\hat{\beta} \operatorname{Ln}(i)$ and $\hat{z}^{r}(i)=\hat{\chi}+\hat{\gamma} i$ are tendency functions with respect to the number of cycles, and $e$ are the error terms which are assumed 
to be independent. The main idea is to compare the accuracies of the two models. If the description (2) improves the estimation of the physical quantity $h$ as compared to the description (3), that means that $z$ causes $h$, since $z$ is an explicative variable of $h$. The non causality hypothesis is expressed by $H_{0}: b_{1}=b_{2}=\ldots=b_{l}=0$. Henceforth, the term " $H_{0}$ " will be used to refer to the above mentioned hypothesis. When this hypothesis is valid, the first term of the system (2) is reduced to the first term of (3). Therefore, it is possible to calculate the causality measure of $z$ and $h$ based on the above mentioned autoregressive models as follows:

$$
\mathcal{F}=(N-2 l-2) \operatorname{Ln}\left(\frac{\Sigma_{e_{s}}}{\Sigma_{\epsilon_{s}}}\right)
$$

where $\mathrm{N}$ denotes the length of the times series, $\Sigma_{e_{s}}$ and $\Sigma_{\epsilon_{s}}$ represent the auto-variances of $e_{s}$ and $\epsilon_{s}$ where $s=h, z$. Under the nullity hypothesis $H_{0}$, the measure $\mathcal{F}_{z \rightarrow h}$ has an asymptotic $\chi^{2}(2 l)$ distribution [11]. In order to reject the hypothesis $H_{0}$ with a preselected risk level $\alpha$, the calculated $\chi^{2}$ should be higher than the value of the standard distribution of parameters $\alpha$ and $l$ ).

\subsection{Estimation coefficients}

The causality measure between the considered signals can be calculated using the error terms $\epsilon_{s}$ and $e_{s}$, where $s=h, z$. Therefore, it is necessary to estimate first of all the coefficients of the suggested models and deduce the error terms. This task can be accomplished using the recursive least square method [4]. In this section, we adapt our description to the algorithm by expressing the signals under consideration as follows:

$d(i)=\boldsymbol{A}^{t} \boldsymbol{x}^{(i)}+u(i)$

where $d(i)$ is an output corresponding to $h, z$ or both of them as will be seen later on, $\boldsymbol{A}$ is a vector which encloses the unknown estimation parameters and $u(i)$ represents the estimation error at the step $i$. In the case of model (2), these vectors can be written as an input $\boldsymbol{x}^{(i)}=[1, \operatorname{Ln}(i), h(i-1), . ., h(i-$ $l), 1, i, z(i-1), . ., z(i-l)]$, an unknown $\boldsymbol{A}=\left[\alpha, \beta, a_{1}, . ., a_{l}, \chi, \gamma, b_{1}, . ., b_{l}\right]$ and error terms $u(i)=\epsilon_{h}(i)$. Similarly, in the case of model (3), these vectors can be written as $\boldsymbol{x}^{(i)}=[1, \operatorname{Ln}(i), h(i-1), . ., h(i-l)], \boldsymbol{A}=\left[\alpha, \beta, a_{1}, . ., a_{l}\right]$ and $u(i)=e_{h}(i)$. In order to estimate the unknown parameters of the model (5), the least squares criterion is used:

$\mathcal{R}=\sum_{i=0}^{n} \lambda^{n-i}(u(i))^{2}$

Solving the problem consists in minimizing the quantity $\mathcal{R}$ with respect to the unknown vector $\boldsymbol{A}$. This leads to a recursive algorithm (appendix), which 
consists in starting from an initial set of parameters $\boldsymbol{A}=\mathbf{0}$, an initial matrix $\boldsymbol{Q}=\boldsymbol{I}$ of size $l \times l$ and a real constant $0<<\lambda<1$. At each iteration $n$, the calculation steps read:

$$
\begin{aligned}
& \boldsymbol{k}=\frac{\lambda^{-1} \boldsymbol{Q x}}{1+\lambda^{-1} \boldsymbol{x} \boldsymbol{Q} \boldsymbol{x}^{t}} \\
& u(n)=d(n)-\boldsymbol{x}^{t} \boldsymbol{A} \\
& \boldsymbol{A}=\boldsymbol{A}+\boldsymbol{k} u(n) \\
& \boldsymbol{Q}=\lambda^{-1}\left(\boldsymbol{Q}-\boldsymbol{k} \boldsymbol{x}^{t} \boldsymbol{Q}\right)
\end{aligned}
$$

where the vector $\boldsymbol{k}$ is termed as the Kalman gain. Knowing the parameters of the models (2) and (3), it is possible to plot out the estimations of the settlement and permanent contacts number as shown in figure (7).

Once the error terms are obtained, the causality measure can be calculated using equation (4). In the particular case considered in this study, the discrete time signals $h$ and $z$ are of length $N=1000$ cycles and the order of autoregression is of $l=N / 100$. The risk level of rejecting the hypothesis $H_{0}$ is $\alpha=1 \%$. At this risk level, and for a number of parameters $l$, the calculated theoretical value of $\chi^{2}(2 l)$ is 37.52 , however, the measure of causality equals 590.85. Therefore, it can be concluded that the number of permanent contacts loss is a significant explication variable of the settlement under sleeper. This approach can be applied for different loading cases. Table (1) shows that the causality direction remains valid for different frequencies and amplitudes of loading.

\section{Conclusion}

In this study, numerical simulations of a partially confined granular material under vibration are presented. It has been shown that the settlement process is characterized by a flow of materials towards less loaded regions. It has also been noticed that there is privileged regions of contacts loss. Furthermore, it has been proved that the loss of contacts causes the settlement, for different loading cases. The residual displacements which take place under dynamic loading at different frequencies and amplitudes depend on several factors such as material properties, grain shape, degree of confinement etc. In this study, we selected the diorite and the polygonal shape because this type of material is widely used in railway platforms. Moreover, we concentrated on the loss of contacts as an explicative variable of settlement. As a perspective of the suggested analysis, it would be interesting to investigate all the physical factors which may influence the settlement speed, such as the acceleration and elastic deflection. It is also possible to extend the suggested procedure for more complex grain shapes. For instance, as long as the settlement depends on the mobility of the material, non convex grains may have an important effect on the settlement. Actually, unlike convex grains (circular, polygonal, elliptic etc.) where the contact are binary, non convex grains can be connected 
with more than a single contact. Assuming equivalent sizes of contact areas, the dissipation should be higher in case of non convex grain. In addition, the contact openings are more difficult to take place, therefore the settlement should be different. This particular point will be the subject of a future contribution by the authors.

\section{A Recursive Least Squares}

Minimizing the least square criterion (6) leads to the following equation:

$\sum_{i=0}^{n} \lambda^{n-i} x(i) \boldsymbol{x}^{(i)}=\sum_{i=0}^{n} \lambda^{n-i}\left[\boldsymbol{x}^{(i)} \boldsymbol{x}^{(i)^{t}}\right] \boldsymbol{A}^{(n)}$

Let $p^{(n)}=\sum_{i=0}^{n} \lambda^{n-i} x(i) \boldsymbol{x}^{(i)}, R^{(n)}=\sum_{i=0}^{n} \lambda^{n-i}\left[\boldsymbol{x}^{(i)} \boldsymbol{x}^{(i)^{t}}\right]$. Using the Woodbury identity of matrices (Kima et Bennighof [17]) one can obtain the following relationship:

$\boldsymbol{Q}^{(n)}=\lambda^{-1} \boldsymbol{Q}^{(n-1)}-\lambda^{-1} \boldsymbol{k}^{(n)} \boldsymbol{x}^{(n)^{t}} \boldsymbol{Q}^{(n-1)}$

where $\boldsymbol{Q}^{(n)}=\boldsymbol{R}^{-\mathbf{1}(n)}$ and $\boldsymbol{k}^{(n)}=\frac{\lambda^{-1} \boldsymbol{Q}^{(n-1) \boldsymbol{x}^{(n)}}}{1+\lambda^{-1} \boldsymbol{x}^{(n)^{t}} \boldsymbol{Q}^{(n-1)} \boldsymbol{x}^{(n)}}$. Introducing this expression in the equation $\boldsymbol{A}^{(n)}=\boldsymbol{Q}^{(n)} \boldsymbol{p}^{(n)}$, leads to a solution of the problem in a recurrent formula:

$\boldsymbol{h}^{(n)}=\boldsymbol{h}^{(n-1)}+\boldsymbol{k}^{(n)} u(n)$

\section{References}

1. Allen M, Tildesley D (1989) Computer Simulation of Liquids. Oxford University Press, Bristol

2. Azema E, Radjai F, Peyroux R, Dubois F, Saussine G (2006) Vibrational dynamics of confined granular material. Physical Review E 74:031,302-031,312

3. Barksdale RD (1972) Laboratory evaluation of rutting in base course materials. Third International Conference on Structural Design of Asphalt Pavement, September 1972, London, England 3:161-174

4. Beex AA, Zeidler JR (2003) Non-wiener effects in recursive least squares adaptation. IEEE pp 7803-7946

5. Chen Y, Rangarajan G, Feng J, Ding M (2004) Analyzing multiple nonlinear time series with extended granger causality. Physics Lettrs A 324:26-35

6. Ciamarra MP, Coniglio A, Nicodemi M (2005) Shear-induced segregation of a granular mixture under horizontal oscillation. Journal of Physics Condensed Matter 17(24):2549-2556

7. Clément E, Rajchenbach J, Duran J (1995) Mixing of a granular material in a bidimensional rotating drum. Materials Research Society Symposium Proceedings 367(13):513-517

8. Combe G, Roux JN (2000) Strain versus stress in a model granular material: A devil's staircase. Physical Review Letters 85(17):3628-3631

9. Cundall PA, Strack ODL (1979) A discrete numerical model for granular assemblies. Géotechnique 29:47-65 
10. Dufour J, Pelletier D, Renault E (2007) Multivariate out-of-sample tests for granger causality. Computational Statistics and Data Analysis 51:3319-3329

11. Gandolfo G, Nicolettiy G (2002) Exchange rate volatility and economic openness: A causal relation ? Centro ricerche di economia internazionale 68

12. Granger C (1969) Investigating causal relations by econometric models and cross -spectral methods. Econometrica 37:424-459

13. Karrech A (2007) Comportement des matériaux granulaires sous vibration: Application au cas du ballast. PhD thesis, Ecole Nationale des Ponts et Chaussées, France

14. Karrech A, Duhamel D, Bonnet G, Canou J, Dupla JC, Roux JN, Chevoir F (2006) Experimental study of settlement mechanisms in micro-ballast beds under dynamic loading. Submitted to the Journal of Materials in Civil Engineering

15. Karrech A, Duhamel D, Bonnet G, Roux JN, Chevoir F, Sab K, Canou J, Dupla JC (2006) Discrete element method for granular materials preparation and settlement. The Third International Conference on Advances In Mechanical Engineering and Mechanics, Hammamet, Tunisia, December 17-19

16. Karrech A, Duhamel D, Bonnet G, Roux JN, Chevoir F, Canou J, Dupla JC, Sab K (2007) A computational procedure for the prediction of settlement in granular materials under cyclic loading. Computer Methods in Applied Mechanics and Engineering 197:80-94

17. Kima CW, Bennighof J (2006) Fast frequency response analysis of partially damped structures with non-proportional viscous damping. Journal of Sound and Vibration 297:1075-1081

18. Lobo-Guerrero S, Vallejo L (2006) Discrete element method analysis of railtrack ballast degradation. Granular Matter 8:195-204

19. Lu M, McDowell GR (2007) The importance of modelling ballast particle shape in the discrete element method. Granular Matter 9:69-80

20. Moon SJ, Goldman DI, Swinney HL, Swift JB (2003) Kink-induced transport and segregation in oscillated granular layers. Physical Review Letters 91(13):1343,011-1343,014

21. Nowak ER, Knight JB, Ben-Naim E, Jaeger HM, Nagel SR (1998) Density fluctuations in vibrated granular materials. Physical Review E Statistical Physics 57(2):1971-1982

22. Ribiere P, Richard P, Bideau D, Delannay R (2005) Experimental compaction of anisotropic granular media. The European Physical Journal E 16:415-420

23. Rosato AD, Doris Y (2000) Microstructure evolution in compacted granular beds. Powder Technology 109:255-261

24. Roux JN, Chevoir F (2005) Simulation numérique discrète et comportement mécanique des matériaux granulaires. Bulletin des Laboratoires des Ponts et Chaussées (254):109-138

25. Saussine G, Cholet C, Gautier P, Dubois F, Bohatier C, Moreau J (2005) Modelling ballast behavior under dynamic loading, part1: A 2d polygonal discrete element method approach. Computer Method in Applied Mechanics and Enginnering 195(19-22):2841-2859

26. Shenton M (1978) Deformation of Railway Ballast under Repeated Loading Conditions. Oxford 
List of Tables

1 Causality measure for different excitations . . . . . . . . . . 13 


\section{List of Figures}

1 (a) Typical grain characterized by $r_{1}, r_{2}$, and $\theta$, (b) Contact detecting using the dichotomy method. . . . . . . . . . . . 14

2 (a) Boundary conditions and applied force (b) Residual displacement of grain (the colorbar represents the intensity of residual displacement in the y-direction in $\mathrm{cm}$ ). . . . . . . . . . . . 15

3 Settlement speed with respect to acceleration. . . . . . . . . . . 16

4 Response of the sample in terms of settlement $\left(h_{\max }\right)$ and number of contacts $\left(Z_{\tau>\Delta t}\right)$ with respect to the number of cycles at different frequencies. ............................ 17

5 Distribution of (a) coordination number (b) material density. . . . . 18

6 Variation of the settlement and of the number of permanent contacts with respect to the number of cycles. . . . . . . . . . . . . 19

7 Application of the models (2) and (3) on the signals of settlement and permanent contacts. The subscripts "in", "1", and " 2 " denote the MD method calculations, the estimation using the first model and the estimation using the second model, respectively. . . . . . . . 20 


\begin{tabular}{lcc}
\hline \hline Frequency, $f(\mathrm{~Hz})$ & Applied Force, $\Delta F(\mathrm{kN})$ & $\chi^{2}$ \\
\hline \multirow{2}{*}{20} & 0.5 & 621.57 \\
& 1 & 227.61 \\
& 1.5 & 457.06 \\
30 & 0.5 & 634.34 \\
& 1 & 750.91 \\
40 & 1.5 & 640.1 \\
& 0.5 & 703.2 \\
& 1 & 430.9 \\
& 1.5 & 685.42 \\
\hline
\end{tabular}

Table 1 Causality measure for different excitations 


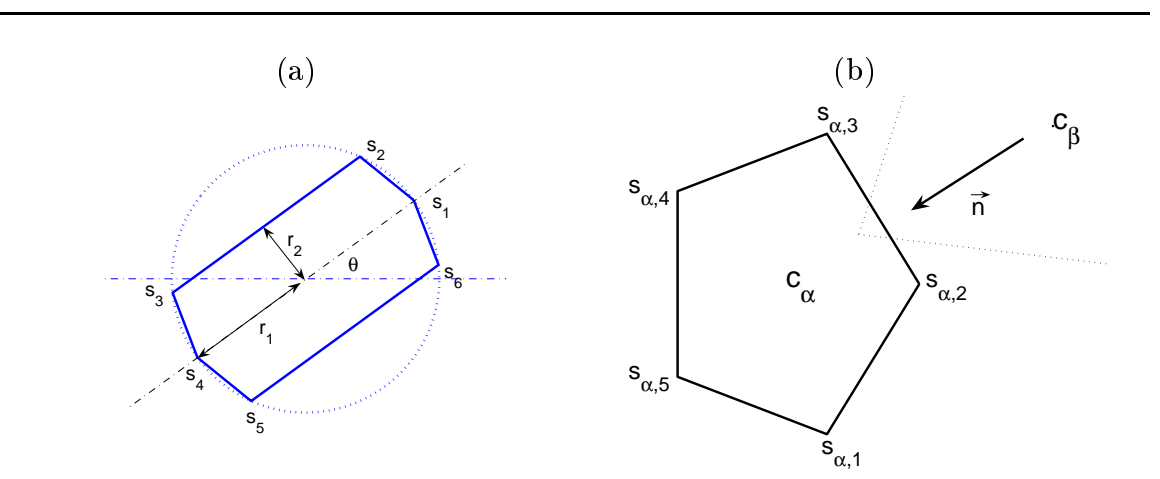

Fig. 1 (a) Typical grain characterized by $r_{1}, r_{2}$, and $\theta$, (b) Contact detecting using the dichotomy method. 
(a)

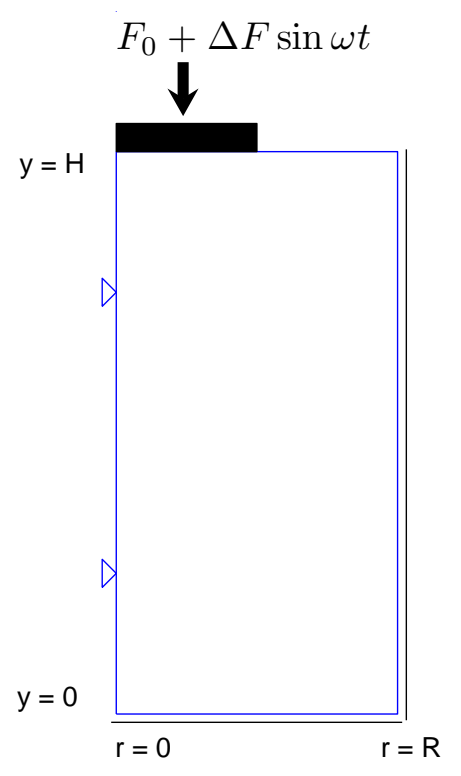

(b)

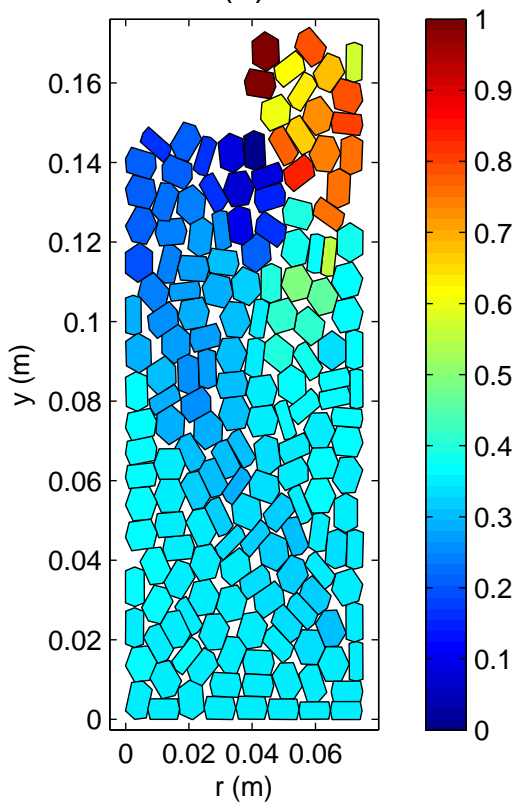

Fig. 2 (a) Boundary conditions and applied force (b) Residual displacement of grain (the colorbar represents the intensity of residual displacement in the $y-$ direction in $\mathrm{cm}$ ). 


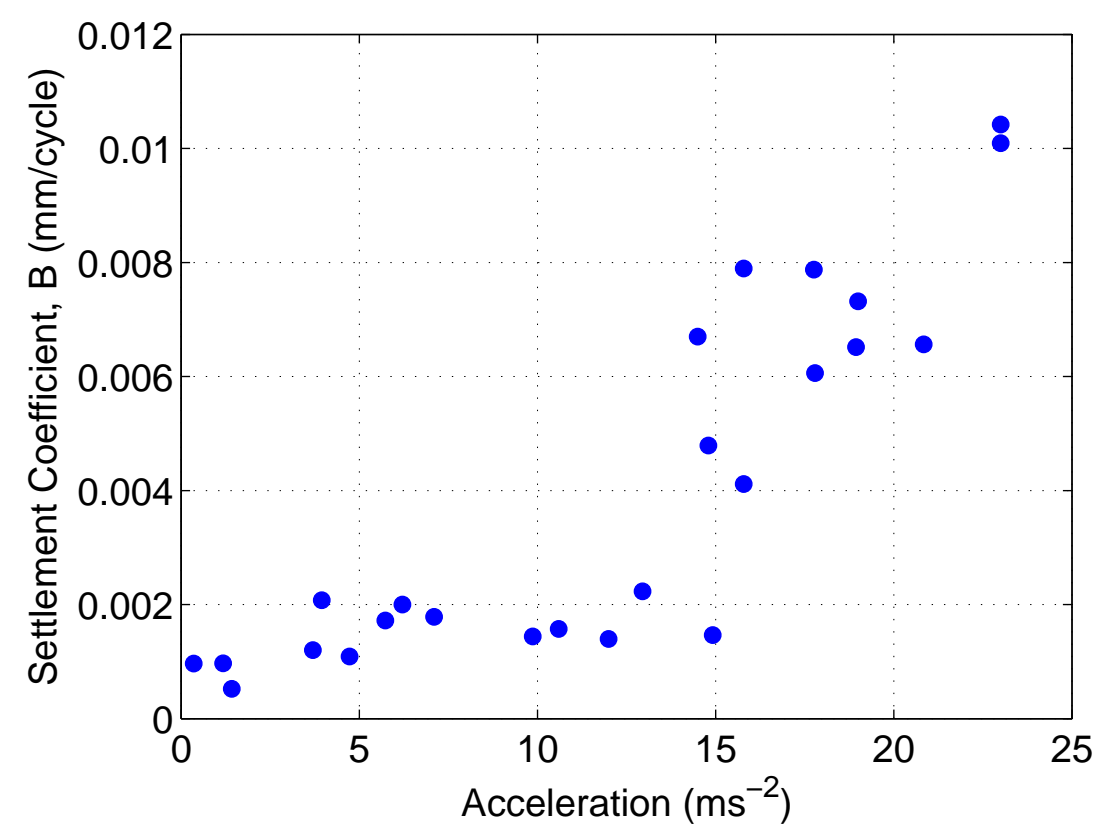

Fig. 3 Settlement speed with respect to acceleration. 

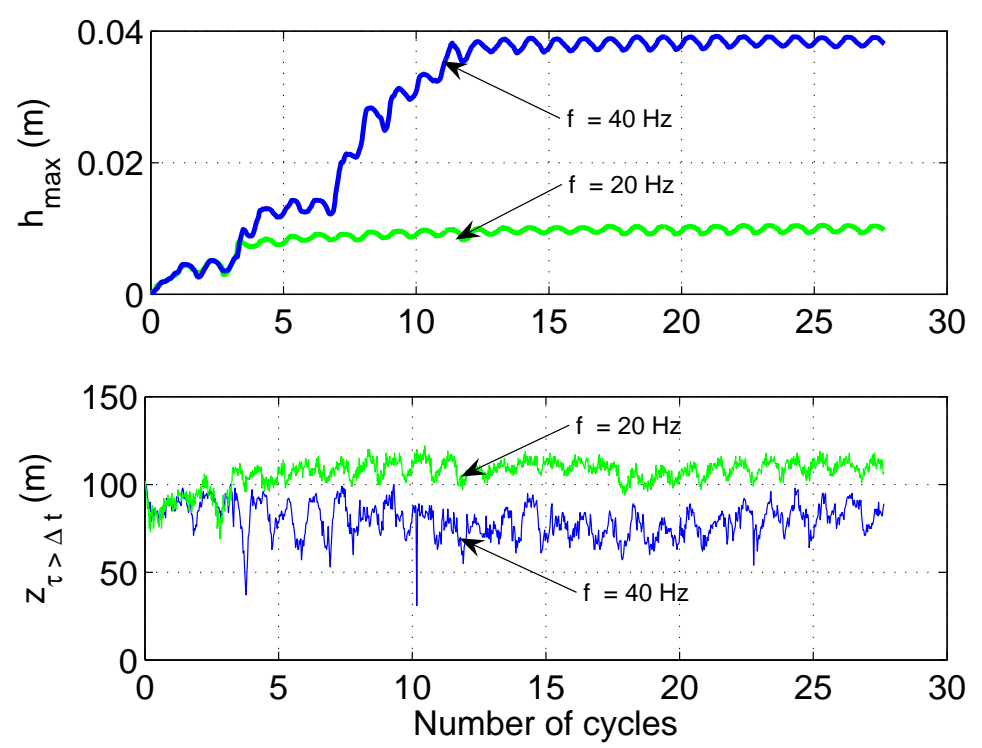

Fig. 4 Response of the sample in terms of settlement $\left(h_{\max }\right)$ and number of contacts $\left(Z_{\tau>\Delta t}\right)$ with respect to the number of cycles at different frequencies. 
(a)

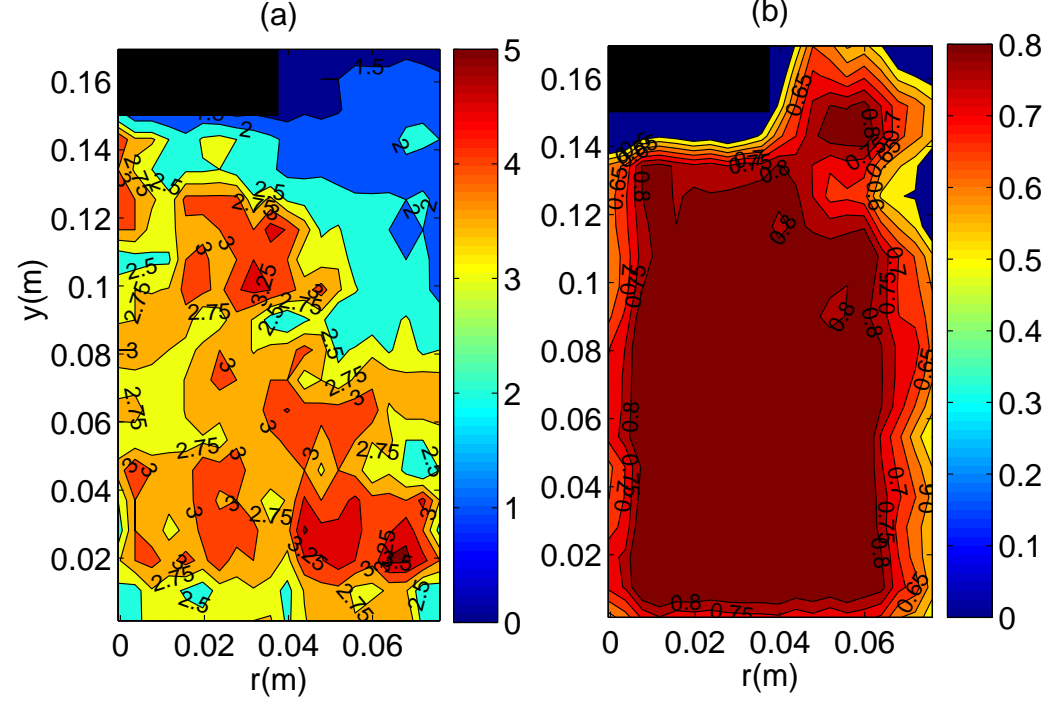

Fig. 5 Distribution of (a) coordination number (b) material density. 


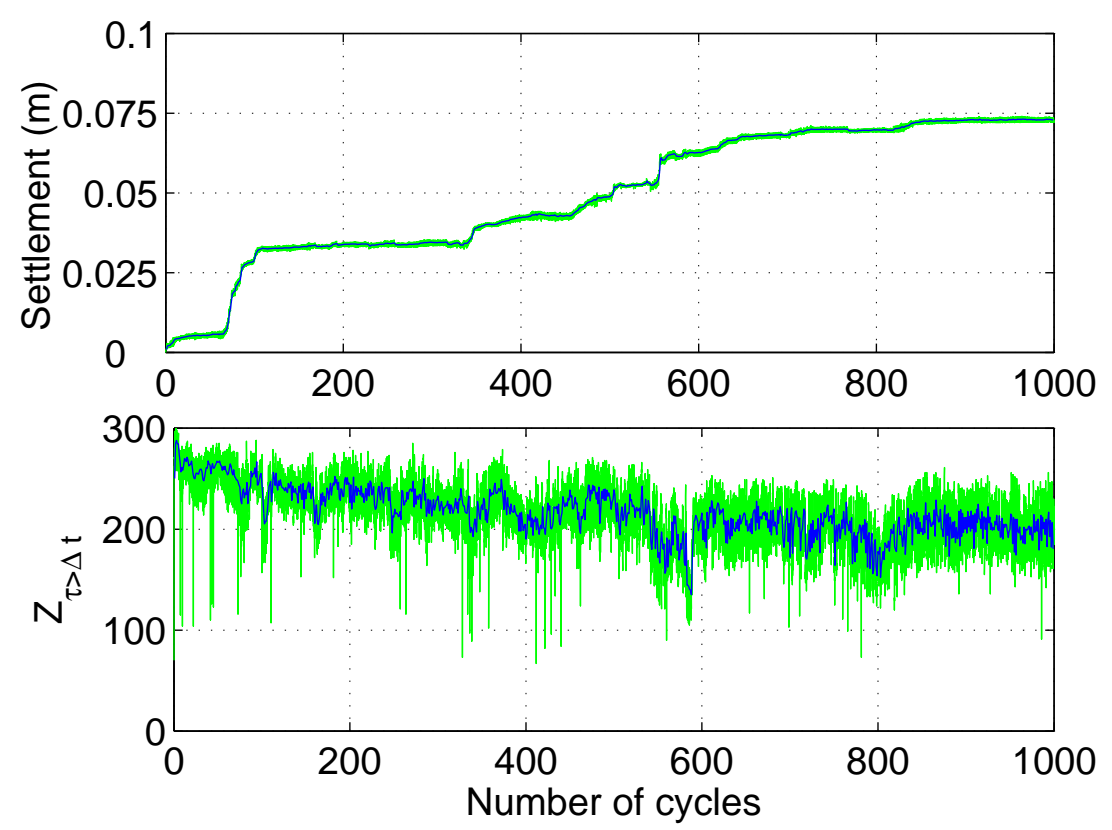

Fig. 6 Variation of the settlement and of the number of permanent contacts with respect to the number of cycles. 


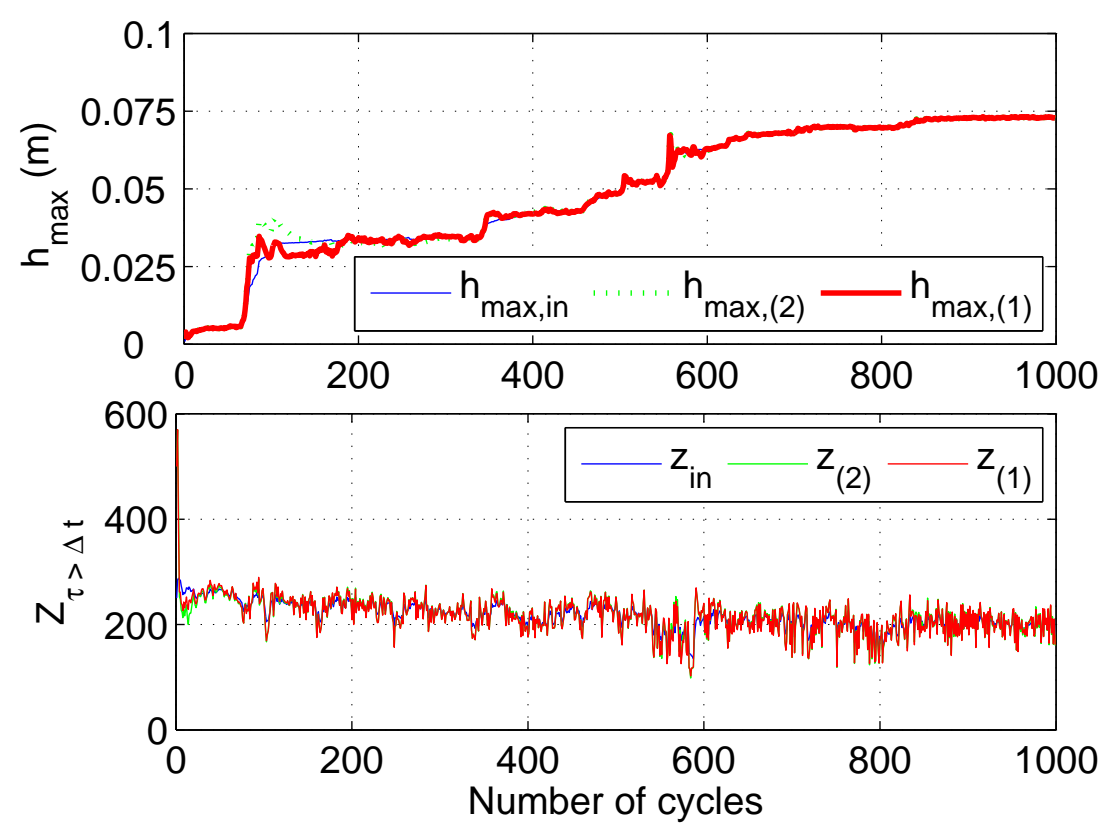

Fig. 7 Application of the models (2) and (3) on the signals of settlement and permanent contacts. The subscripts "in", "1", and "2" denote the MD method calculations, the estimation using the first model and the estimation using the second model, respectively. 